\title{
Myrciaria dubia (Kunth) McVaugh (Myrtales: Myrtaceae): análise dos produtos elaborados a partir do seu fruto
}

\section{Carlena Sinara Martins da Silva ${ }^{1,2}$ e Rosa Helena Veras Mourão $^{2}$}

${ }^{1}$ Universidade Federal do Pará. Instituto de Ciências Biológicas. Programa de Pós-Graduação em Biodiversidade e Biotecnologia. Rua Augusto Corrêa, 1. BelémPA, Brasil (CEP 66075-110). E-mail: karlena_sinara@hotmail.com.

${ }^{2}$ Universidade Federal do Oeste do Pará. Instituto de Saúde Coletiva. Laboratório de Bioprospecção e Biologia Experimental. Rua Vera Paz, S/№. Salé. Santarém-PA, Brasil (CEP 68040-070).

Resumo. Myrciaria dudia, também conhecida como camu-camu, é uma planta que tem ocorrência espontânea na Amazônia e vem ganhando destaque devido aos avanços ocorridos na área científica e na busca da população por terapias e alimentos mais saudáveis. 0 objetivo deste artigo foi realizar um levantamento bibliográfico sobre produtos oriundos de Myrciaria dubia. 0 levantamento foi realizado em plataformas on line lens.org, Instituto Nacional da Propriedade Industrial (INPI) e na base de dados do Portal Coordenação de Aperfeiçoamento de Pessoal de Nível Superior (CAPES), bem como de produtos comercializados na plataforma de busca Google. Os parâmetros de busca utilizados foram os termos "Myrciaria dubia" ou "camu-camu", com intervalo temporal de busca entre 01/01/1990 e 10/05/2019. Os registros de patentes mais depositados para uso de Myrciaria dubia estão relacionados a cosméticos principalmente com ação antioxidante para a pele. Dos 21 pedidos de patentes, apenas quatro são de patentes concedidas. Sobre os artigos científicos, dos 212 relacionados com a espécie, nove referiam-se a desenvolvimento de produtos em publicações de 2003 a 2019, onde a revista que mais publicou foi a Food Science and Technology. Dentre os produtos encontrados nas publicações observa-se um avanço para a área de alimentos e cosméticos. No que se refere aos produtos comercializados pela Internet, exeste uma variação de produtos como na forma de pó (19), cápsulas (14), licor (3), chocolate (1), geleia (1) e muda (1). 0 valor dos produtos variando entre $\mathrm{R} \$ 12,50$ e $\mathrm{R} \$ 199,00$. Observamos que os produtos oriundos de Myrciaria dubia estão sendo difundidos e vem crescendo devido principalmente ao seu alto teor de bioativos com pespectivas de uso pelas indústrias de alimentos, cosméticos e farmacêuticas.

Recebido

$08 / 08 / 2021$

Aceito

$24 / 12 / 2021$

Publicado

$31 / 12 / 2021$

Acesso aberto

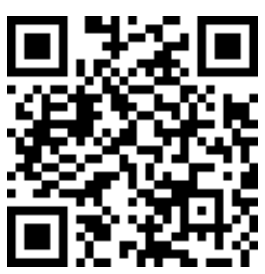

ORCID

(D) $0000-0002-1543-8334$ Carlena Sinara Martins da Silva

(D) 0000-0003-0100-644X Rosa Helena Veras Mourão

ISSN 2359-1412/RBGAS-2021-0097/2021/8/20/26/1687

Rev. Bras. Gest. Amb. Sustent.

http://revista.ecogestaobrasil.net 
Palavras-chave: Myrciaria dubia; Camu-camu; Comércio; Produtos.

\begin{abstract}
Myrciaria dubia (Kunth) McVaugh (Myrtales: Myrtaceae): Analysis of the products made from its fruit. Myrciaria dudia, also known as camu-camu, is a plant that occurs spontaneously in the Amazon and has gained prominence due to advances in the scientific area and in the population's search for healthier therapies and foods. The objective of this work was to carry out a bibliographical survey on products from Myrciaria dubia. The survey was carried out on online platforms lens.org, Brazilian National Institute of Industrial Property (INPI) and on the database of the Brazilian Coordination for the Improvement of Higher Education Personnel (CAPES), as well as products sold on the google search platform. The search parameters used were the terms "Myrciaria dubia" or "camu-camu", with a search time interval between Jan. 01, 1990 and May 10, 2019. The most filed of patents for the use of Myrciaria dubia are related to cosmetics mainly with antioxidant action for the skin. Of the 21 patent applications, only 4 are granted patents. Of the 212 scientific articles related to the species, 9 referred to product development in publications from 2003 to 2019 , where the magazine that published the most was Food Science and Technology. Among the products found in the publications, there is an advance in the food and cosmetics area. With regard to products sold on the internet, the most sold were powder (19), capsules (14), liquor (3), chocolate (1), jelly (1) and seedling (1). The value of products ranging from $R \$ 12.50$ to $R \$ 199.00$. We observe that the products from Myrciaria dubia are being disseminated and are growing mainly due to their high content of bioactives with perspectives of use by the food, cosmetics and pharmaceutical industries.
\end{abstract}

Keywords: Myrciaria dubia; Camu-camu; Business; Products.

\title{
Introdução
}

A Myrciaria dubia (Kunth) McVaugh (Myrtales: Myrtaceae), conhecida como camu-camu, camocamo, caçari, araçá-d'água, araçá-de-igapó, guayabo e guayabito, é uma espécie de porte arbustivo encontrada às margens dos rios, lagos, região de várzea e igapó da Amazônia (Arruda et al., 2011). Tem ocorrência natural no Peru, Brasil, Venezuela e Colômbia (Chagas et al., 2015). No Brasil, tem ocorrências confirmadas no Acre, Amazonas, Pará, Rondônia, Roraima e Mato Grosso (Sobral et al., 2014).

0 fruto do camu-camu, por apresentar elevado teor de ácido ascórbico, geralmente superior a $2 \mathrm{~g} \times 100 \mathrm{~g}^{-1}$ (Morais e Pinheiro , 2018) e de outros princípios ativos como antocianinas, com teores médios de $54 \mathrm{mg}$ por $100 \mathrm{~g}$ (Rodrigues e Marx, 2006; Grigo et al., 2021), tem despertando o interesse de diversas indústrias nacional e internacional, devido à grande demanda de utilização de produtos naturais sejam nos setores alimentício, farmacêutico ou cosméticos. Nos EUA e no Japão a $M$. dubia é utilizada na indústria 
farmacêutica e na forma de produtos energéticos e nutracêuticos e em alguns países da Europa é utilizada a polpa da fruta seca, extrato em pó e cápsulas, ou como parte de suplementos alimentares e no Peru usado no preparo de sucos, geleias, sorvetes e doces (Ribeiro et al., 2006; Santos et al., 2018).

Na Região Norte do Brasil, os frutos de M. dubia são coletados diretamente da planta quando inicia o processo de amadurecimento, observando a coloração da casca que varia de vermelho ou róseo a roxo escuro, sendo usados no preparo de refresco, sorvete, picolé, geleia, xarope, doce e licores. É uma das espécies requeridas para compor sistemas agroflorestais na Amazônia Central, pois ainda não existe um sistema de plantação em escala comercial que atenda à demanda internacional (Viégas et al., 2004; Chagas et al., 2012; Billacrês et al., 2020).

0 fruto de camu-camu tem potencial para ser usado no desenvolvimento de novos alimentos ricos em bioativos e, possivelmente, na eliminação do uso de conservantes químicos sintéticos em alimentos processados. Por ser uma espécie que tem ocorrência espontânea na Amazônia, vem ganhando destaque devido aos avanços ocorridos na área científica e na busca da população por terapias e alimentos mais saudáveis. Sendo assim, o objetivo deste estudo foi realizar um levantamento bibliográfico sobre produtos oriundos de Myrciaria dubia por meio de compilação de artigos científicos, patentes e produtos clmercializados.

\section{Material e métodos}

\section{Produtos originados de Myrciaria dubia: patentes}

A prospecção foi realizada com base nos pedidos de patentes depositadas nas bases internacionais e nacionais, através do uso da plataforma on line lens.org (https://www.lens.org/) e diretamente nas ferramentas de busca do Instituto Nacional da Propriedade Industrial (INPI) (https://busca.inpi.gov.br/pePI/jsp/patentes/), respectivamente.

Os parâmetros de busca utilizados foram o termo de busca: "Myrciaria dubia" ou "camu-camu", o intervalo temporal da busca foram pedidos de patentes entre 01/01/1990 e 10/05/2019, sendo analisados todos os pedidos de patentes cujas termos de busca apresentavam no título e/ou resumo dos pedidos.

\section{em base de dados \\ Desenvolvimento de produtos a partir de Myrciaria dubia: artigos científicos \\ Nas bases de dados Portal Coordenação de Aperfeiçoamento de Pessoal de Nível} Superior (CAPES) (http://www.capes.gov.br), realizou-se busca de publicações originalmente na língua inglesa e portuguesa. Os parâmetros de busca utilizados foram o termo de busca: "Myrciaria dubia" ou "camu-camu", o intervalo temporal da busca foram pesquisados pedidos de patentes entre 01/01/2003 e 10/05/2019, sendo analisados todos aos artigos cujo termo de busca se apresentassem no título e/ou resumo dos pedidos.

\section{Produtos comercializados com Myrciaria dubia}

Para realizar o levantamento de dados dos produtos mais comercializados derivados de Myrciaria dubia, foi usado como descritor o nome popular da espécie vegetal camu-camu, na plataforma de busca Google (https://www.google.com.br/), em 2019.

\section{Resultados e discussão}

Produtos originados de Myrciaria dubia: patentes 
$\mathrm{Na}$ busca dos registos na plataforma lens.org, resultou em vasto volume de informações (241 patentes). As publicações que tinham relação com o objetivo da pesquisa, ou seja, produtos provenientes do fruto da espécie foram catalogadas (13 patentes) publicadas de 2006 a 2019. Já na plataforma INPI foram encontradas oitp pedidos.

Os requerentes que mais tem patentes na plataforma lens.org, foram a Nichirei Biosciences Inc. (2) e Mary Kay Inc. (2), e no INPI, foram o Instituto Nacional de Pesquisas da Amazônia (INPA) da Amazônia (3) e Rondônia (3) (Tabela 1).

Tabela 1. Patentes que foram requeridas com produtos provenientes do fruto Myrciaria dubia.

\begin{tabular}{|l|c|c|c|}
\hline Requerente & Ano de publicação & $\begin{array}{c}\text { Quantidade de } \\
\text { publicações por } \\
\text { requerentes }\end{array}$ & $\begin{array}{c}\text { Plataforma } \\
\text { de busca }\end{array}$ \\
\hline Nichirei Biosciences Inc & $2006 / 2010$ & 2 & lens.org \\
\hline Smith Walter P & 2009 & 1 & lens.org \\
\hline Mazed Mohammad A; Mazed Sayeeda & 2010 & 1 & lens.org \\
\hline $\begin{array}{l}\text { Pharmabrand S A; Cid Vivanco } \\
\text { Roberto Francisco; Andrade Bejarano } \\
\text { Edwin Renato }\end{array}$ & 2013 & 1 & lens.org \\
\hline San Juan Amazonia Europa S L & 2014 & 1 & lens.org \\
\hline Mary Kay Inc & $2015 / 2018$ & 2 & lens.org \\
\hline Parham Tabibian MD Inc & 2017 & 1 & lens.org \\
\hline Stewart Lisa; Asadorian Ara & 2018 & 1 & lens.org \\
\hline $\begin{array}{l}\text { Isp Investments LLC; Jafer Enterprises } \\
\text { R\&D S L Soc Unipersonal }\end{array}$ & 2019 & 1 & lens.org \\
\hline Easterling John Harvey & 2019 & 1 & lens.org \\
\hline $\begin{array}{l}\text { Instituto Nacional de Pesquisas da } \\
\text { Amazônia - INPA (BR/AM) }\end{array}$ & 2013 & 3 & INPI \\
\hline Plantextrakt GMBH \& Co. KG (DE) & $2016 / 2019$ & 1 & INPI/lens.org \\
\hline $\begin{array}{l}\text { Instituto Federal de Educação, Ciência } \\
\text { e Tecnologia de Rondônia (BR/RO) }\end{array}$ & 2019 & 3 & INPI \\
\hline
\end{tabular}

Fonte: Plataforma lens.org e INPI.

Dentre os setores que tem crescimento expressivo e investimento em pesquisas e desenvolvimento de novos produtos, a indústria cosmética e de produtos para higiene pessoal, apresenta grande importância para a economia brasileira (Garcia, 2005). 0 que corroboram com os resultados encontrados na pesquisa, onde os registros de patentes mais depositados do uso do fruto de Myrciaria dubia foi em cosméticos (10), publicados entre 2010 e 2019, onde apenas quatro dos 21 registros são de patentes concedidas (Tabela 2).

As revidicações das patentes estão voltadas majoritariamente para o uso cosmético (10). Nagamine et al. $(2006,2010)$ desenvolveu uma formulação de uso externo com atividade antioxidante e efeito de clareamento. Além disso, o extrato de polpa e pele de frutos secos e sem sementes de Myrciaria dubia, foram usados com o intuito de reduzir os sinais de fadiga da pele (Botto et al., 2019). A combinação de quantidades de extratos de Malpighia punicifolia (acerola), Myrciaria dubia (camu-camu) e Ribes nigrum (groselha preta), foram suficientes para aumentar a produção de laminina na pele, constituinte da 
matriz extracelular atuando na adesão de células epiteliais à sua lâmina basal, auxiliando na firmeza da pele (Hines e Florence, 2015).

Tabela 2. Patentes com produtos oriundos de Myrciaria dubia.

\begin{tabular}{|c|c|c|c|c|c|}
\hline Produto & $\begin{array}{l}\text { Parte da planta / } \\
\text { tipo de extrato }\end{array}$ & Classificação & Inventor & $\begin{array}{l}\text { Ano de } \\
\text { publ. }\end{array}$ & Tipo \\
\hline $\begin{array}{l}\text { Composição de } \\
\text { antioxidantes }\end{array}$ & $\begin{array}{l}\text { Fruto sem semente, } \\
\text { secando as frutas } \\
\text { sem sementes a uma } \\
\text { temperatura }<60^{\circ} \mathrm{C}\end{array}$ & Cosmético & $\begin{array}{c}\text { Iranzo Pous } \\
\text { Gonzalo; Milan } \\
\text { Navarro } \\
\text { Susana } \\
\end{array}$ & 2014 & $\begin{array}{l}\text { Pedido de } \\
\text { Patente }\end{array}$ \\
\hline $\begin{array}{l}\text { Composição } \\
\text { para um } \\
\text { tratamento } \\
\text { antienvelhecim } \\
\text { ento }\end{array}$ & $\begin{array}{l}\text { Composição } \\
\text { contendo pelo } \\
\text { menos } 100-500 \mathrm{mg} \\
\text { de camu-camu }\end{array}$ & Cosmético & $\begin{array}{l}\text { Tabibian } \\
\text { Parham } \\
\text { Michael }\end{array}$ & 2017 & $\begin{array}{l}\text { Patente } \\
\text { Concedida }\end{array}$ \\
\hline $\begin{array}{l}\text { Composto, } \\
\text { processo para } \\
\text { produzir e } \\
\text { usá-lo }\end{array}$ & $\begin{array}{l}\text { Suco de fruta de } \\
\text { camu-camu }\end{array}$ & Cosmético & $\begin{array}{c}\text { Nagamine } \\
\text { Kenichi; } \\
\text { Hayashi } \\
\text { Mik;Yamasaki } \\
\text { Kaori } \\
\end{array}$ & 2006 & $\begin{array}{c}\text { Patente } \\
\text { Concedida }\end{array}$ \\
\hline $\begin{array}{c}\text { Método para a } \\
\text { preparação de } \\
\text { um extrato seco } \\
\text { e pouco } \\
\text { higroscópico }\end{array}$ & $\begin{array}{c}\text { Extrato seco de } \\
\text { baixa higroscopia de } \\
\text { uma fruta rica em } \\
\text { vitamina } \mathrm{C} \text { ou } \\
\text { espécies vegetais, } \\
\text { como Acerola } \\
\text { (Malpighia glabra), } \\
\text { Camu-camu } \\
\text { (Myrciaria dubia) ou } \\
\text { semelhantes } \\
\end{array}$ & $\begin{array}{l}\text { Técnica de } \\
\text { extração }\end{array}$ & $\begin{array}{c}\text { Plantextrakt } \\
\text { GMBH \& Co. KG }\end{array}$ & 2019 & $\begin{array}{l}\text { Patente } \\
\text { Concedida }\end{array}$ \\
\hline $\begin{array}{c}\text { Métodos e } \\
\text { composições } \\
\text { para reduzir a } \\
\text { aparência de } \\
\text { rugas faciais } \\
\text { dinâmicas (oral } \\
\text { ou tópico) }\end{array}$ & $\begin{array}{l}\text { Composição } \\
\text { contendo extrato do } \\
\text { fruto }\end{array}$ & Cosmético & Smith Walter P & 2009 & $\begin{array}{l}\text { Pedido de } \\
\text { Patente }\end{array}$ \\
\hline $\begin{array}{c}\text { Métodos e } \\
\text { composições } \\
\text { para o } \\
\text { tratamento de } \\
\text { estrias distesas } \\
\end{array}$ & $\begin{array}{l}\text { Composição com } \\
\text { extrato de frutas } \\
\text { Myrciaria dubia }\end{array}$ & Cosmético & $\begin{array}{l}\text { Carle Tiffany; } \\
\text { Kalahasti } \\
\text { Geetha; Burkes } \\
\text { Shona; Gan } \\
\text { David } \\
\end{array}$ & 2018 & $\begin{array}{l}\text { Pedido de } \\
\text { Patente }\end{array}$ \\
\hline $\begin{array}{l}\text { Extratos ricos } \\
\text { em ácidos } \\
\text { orgânicos, } \\
\text { composições } \\
\text { cosméticas que } \\
\text { incluem os } \\
\text { mesmos usos e } \\
\text { cosméticos }\end{array}$ & $\begin{array}{l}\text { Polpa com casca de } \\
\text { frutos secos de } \\
\text { Myrciaria dubia, sem } \\
\text { sementes }\end{array}$ & Cosmético & $\begin{array}{c}\text { Botto Jean- } \\
\text { Marie; } \\
\text { Capallere } \\
\text { Christophe;Go } \\
\text { ndran } \\
\text { Catherine; } \\
\text { Imbert } \\
\text { Isabelle; } \\
\text { Garnier } \\
\text { Sébastien; Le } \\
\text { Borgne erell } \\
\end{array}$ & 2019 & $\begin{array}{l}\text { Pedido de } \\
\text { Patente }\end{array}$ \\
\hline
\end{tabular}


Tabela 2. Continuação.

\begin{tabular}{|c|c|c|c|c|c|}
\hline Produto & $\begin{array}{l}\text { Parte da planta / } \\
\text { tipo de extrato }\end{array}$ & Classificação & Inventor & $\begin{array}{l}\text { Ano de } \\
\text { publ. }\end{array}$ & Tipo \\
\hline $\begin{array}{c}\text { Composição } \\
\text { Antioxidante } \\
\text { Natural } \\
\text { Antioxidante }\end{array}$ & $\begin{array}{c}\text { Composição de } \\
\text { extrato de Myrciaria } \\
\text { dubia e Echinacea } \\
\text { purpúrea }\end{array}$ & Cosmético & $\begin{array}{l}\text { Cid Vivanco } \\
\text { Roberto } \\
\text { Francisco; } \\
\text { Andrade } \\
\text { Bejarano } \\
\text { Edwin Renato }\end{array}$ & 2013 & $\begin{array}{l}\text { Pedido de } \\
\text { Patente }\end{array}$ \\
\hline $\begin{array}{l}\text { Suplemento } \\
\text { nutricional para } \\
\text { prevenção de } \\
\text { doenças } \\
\text { cardiovasculare } \\
\text { s, doenças de } \\
\text { Alzheimer, } \\
\text { diabetes e } \\
\text { regulação e } \\
\text { redução de } \\
\text { açúcar no } \\
\text { sangue e } \\
\text { resistência à } \\
\text { insulina }\end{array}$ & $\begin{array}{c}\text { Compreende } \\
\text { qualquer } \\
\text { componente em } \\
\text { qualquer forma de } \\
\text { qualquer parte da } \\
\text { planta }\end{array}$ & Suplemento & $\begin{array}{c}\text { Mazed } \\
\text { Mohammad A; } \\
\text { Mazed Sayeeda }\end{array}$ & 2010 & $\begin{array}{l}\text { Pedido de } \\
\text { Patente }\end{array}$ \\
\hline $\begin{array}{l}\text { Composição } \\
\text { para melhorar } \\
\text { plantas }\end{array}$ & $\begin{array}{l}\text { Composição um pó } \\
\text { de fruta camu-camu, } \\
\text { um pó de caule e } \\
\text { raiz de Muira puama } \\
\text { e uma raiz de maca }\end{array}$ & $\begin{array}{l}\text { Suplemento } \\
\text { para plantas }\end{array}$ & $\begin{array}{c}\text { Easterling John } \\
\text { Harvey }\end{array}$ & 2019 & $\begin{array}{l}\text { Pedido de } \\
\text { Patente }\end{array}$ \\
\hline $\begin{array}{c}\text { Formulações } \\
\text { tópicas para } \\
\text { cuidados com a } \\
\text { pele }\end{array}$ & $\begin{array}{c}\text { Composição } \\
\text { contendo extrato de } \\
\text { Malpighia punicifolia } \\
\text { (acerola), extrato de } \\
\text { Myrciaria dubia } \\
\text { (camu-camu) e } \\
\text { extrato de Ribes } \\
\text { nigrum (groselha } \\
\text { preta) } \\
\end{array}$ & Cosmético & $\begin{array}{l}\text { HInes Michelle; } \\
\text { Florence } \\
\text { Tiffany }\end{array}$ & 2015 & $\begin{array}{l}\text { Pedido de } \\
\text { Patente }\end{array}$ \\
\hline $\begin{array}{l}\text { Formulações } \\
\text { tópicas para } \\
\text { cuidados com a } \\
\text { pele e métodos } \\
\text { de utilização } \\
\text { dos mesmos }\end{array}$ & $\begin{array}{c}\text { As composições } \\
\text { incluem partículas } \\
\text { de camu-camu não } \\
\text { dissolvidas e } \\
\text { solúveis em água } \\
\text { como um esfoliante } \\
\text { em um veículo } \\
\text { farmaceuticamente } \\
\text { aceitável } \\
\end{array}$ & Cosmético & $\begin{array}{l}\text { Stewart Lisa; } \\
\text { Asadorian Ara }\end{array}$ & 2018 & $\begin{array}{l}\text { Patente } \\
\text { Concedida }\end{array}$ \\
\hline $\begin{array}{c}\text { Agente de } \\
\text { clareamento, } \\
\text { preparação da } \\
\text { pele para uso } \\
\text { externo e } \\
\text { cosméticos } \\
\end{array}$ & $\begin{array}{c}\text { Extrato de semente } \\
\text { de camu-camu como } \\
\text { um componente } \\
\text { ativo }\end{array}$ & Cosmético & $\begin{array}{l}\text { Nagamine } \\
\text { Kenichi; } \\
\text { Hayashi Miki; } \\
\text { Furukawa } \\
\text { Kaori }\end{array}$ & 2010 & $\begin{array}{l}\text { Patente } \\
\text { Concedida }\end{array}$ \\
\hline
\end{tabular}


Tabela 2. Continuação.

\begin{tabular}{|c|c|c|c|c|c|}
\hline Produto & $\begin{array}{l}\text { Parte da planta / } \\
\text { tipo de extrato }\end{array}$ & Classificação & Inventor & $\begin{array}{l}\text { Ano de } \\
\text { publ. }\end{array}$ & Tipo \\
\hline $\begin{array}{c}\text { Fermentado } \\
\text { alcoólico suave } \\
\text { de camu-camu } \\
\text { (Myrciaria } \\
\text { dubia) - vinho } \\
\text { suave de camu- } \\
\text { camu }\end{array}$ & $\begin{array}{l}\text { Polpa do fruto em } \\
\text { fermentação, } \\
\text { realizado por } \\
\text { microrganismos que } \\
\text { convertem } \\
\text { moléculas Bde } \\
\text { carboidratos } \\
\text { (açúcares) em } \\
\text { álcool, gás carbônico } \\
\text { e energia. }\end{array}$ & Bebida & $\begin{array}{c}\text { Instituto } \\
\text { Federal de } \\
\text { Educação, } \\
\text { Ciência e } \\
\text { Tecnologia de } \\
\text { Rondônia } \\
\text { (BR/RO) }\end{array}$ & 2019 & $\begin{array}{l}\text { Pedido de } \\
\text { Patente }\end{array}$ \\
\hline $\begin{array}{c}\text { Cerveja de } \\
\text { camu-camu } \\
\text { (Myrciaria } \\
\text { dubia })\end{array}$ & $\begin{array}{l}\text { Polpa da fruta usada } \\
\text { para obter uma } \\
\text { cerveja suave com } \\
\text { sabor acentuado da } \\
\text { fruta }\end{array}$ & Bebida & $\begin{array}{c}\text { Instituto } \\
\text { Federal de } \\
\text { Educação, } \\
\text { Ciência e } \\
\text { Tecnologia de } \\
\text { Rondônia } \\
\text { (BR/RO) }\end{array}$ & 2019 & $\begin{array}{l}\text { Pedido de } \\
\text { Patente }\end{array}$ \\
\hline $\begin{array}{c}\text { Fermentado } \\
\text { alcoólico seco } \\
\text { de camu-camu } \\
\text { (myrciaria } \\
\text { dubia) - vinho } \\
\text { seco de camu- } \\
\text { camu } \\
\end{array}$ & $\begin{array}{l}\text { Polpa do fruto em } \\
\text { fermentação, } \\
\text { realizado por } \\
\text { Microorganismos }\end{array}$ & Bebida & $\begin{array}{c}\text { Instituto } \\
\text { Federal de } \\
\text { Educação, } \\
\text { Ciência e } \\
\text { Tecnologia de } \\
\text { Rondônia } \\
\text { (BR/RO) } \\
\end{array}$ & 2019 & $\begin{array}{l}\text { Pedido de } \\
\text { Patente }\end{array}$ \\
\hline $\begin{array}{l}\text { Método para a } \\
\text { preparação de } \\
\text { um extrato } \\
\text { seco, de baixa } \\
\text { higoroscopia, } \\
\text { de espécie } \\
\text { vegetal ou fruta } \\
\text { ricas em } \\
\text { vitamina C, } \\
\text { como Malpighia } \\
\text { glabra e } \\
\text { Myrciaria dubia } \\
\text { ou semelhantes }\end{array}$ & $\begin{array}{l}\text { Prepararação de um } \\
\text { suco das espécies, - } \\
\text { remover pelo menos } \\
\text { uma parte dos } \\
\text { açúcares contidos } \\
\text { no suco por um } \\
\text { processo } \\
\text { fermentativo }\end{array}$ & Extrato seco & $\begin{array}{c}\text { Plantextrakt } \\
\text { GMBH \& Co. KG } \\
\text { (DE) }\end{array}$ & $\begin{array}{c}2016 \mathrm{e} \\
2019\end{array}$ & $\begin{array}{c}\text { Patente } \\
\text { Concedida }\end{array}$ \\
\hline $\begin{array}{l}\text { Formulação e } \\
\text { processo de } \\
\text { obtenção do } \\
\text { néctar de baixa } \\
\text { caloria (light) a } \\
\text { partir do fruto } \\
\text { de camu-camu }\end{array}$ & $\begin{array}{l}\text { Obtenção de néctar } \\
\text { de baixa caloria } \\
\text { (light) dietético }\end{array}$ & Bebida & $\begin{array}{c}\text { Instituto } \\
\text { Nacional de } \\
\text { Pesquisas da } \\
\text { Amazônia - } \\
\text { INPA (BR/AM) }\end{array}$ & 2013 & $\begin{array}{l}\text { Pedido de } \\
\text { patente }\end{array}$ \\
\hline
\end{tabular}


Tabela 2. Continuação.

\begin{tabular}{|c|c|c|c|c|c|}
\hline Produto & $\begin{array}{l}\text { Parte da planta / } \\
\text { tipo de extrato }\end{array}$ & Classificação & Inventor & $\begin{array}{l}\text { Ano de } \\
\text { publ. }\end{array}$ & Tipo \\
\hline $\begin{array}{l}\text { Composição } \\
\text { processo de } \\
\text { preparação } \\
\text { baseada em } \\
\text { extratos } \\
\text { vegetais para } \\
\text { redução da } \\
\text { glicemia e/ou } \\
\text { colesterol }\end{array}$ & $\begin{array}{l}\text { Uma composição } \\
\text { baseada em extratos } \\
\text { vegetais para } \\
\text { redução da glicemia } \\
\text { e/ou colesterolemia, } \\
\text { em especial a partir } \\
\text { de frutos cupuaçu } \\
\text { (Theobroma } \\
\text { grandiflorum), } \\
\text { camu-camu } \\
\text { (Myrciaria dubia), } \\
\text { cubiu (Solanum } \\
\text { sessiliflorum) }\end{array}$ & Extrato & $\begin{array}{c}\text { Instituto } \\
\text { Nacional de } \\
\text { Pesquisa da } \\
\text { Amazônia } \\
\text { (BR/AM)/Fund } \\
\text { ação } \\
\text { Universidade } \\
\text { do Amazonas } \\
\text { (BR/AM) }\end{array}$ & 2013 & $\begin{array}{l}\text { Pedido de } \\
\text { Patente }\end{array}$ \\
\hline $\begin{array}{l}\text { Composição } \\
\text { baseada emm } \\
\text { extratos } \\
\text { vegetais com } \\
\text { baixo valor } \\
\text { energético, } \\
\text { processo de } \\
\text { preparo de } \\
\text { composição e } \\
\text { método para } \\
\text { redução de } \\
\text { peso }\end{array}$ & $\begin{array}{c}\text { Uma composição } \\
\text { baseada em extratos } \\
\text { vegetais com baixo } \\
\text { valor energético, em } \\
\text { especial a partir de } \\
\text { frutos cupuaçu } \\
\text { Theobroma } \\
\text { grandiflorum, camu- } \\
\text { camu Myrciaria } \\
\text { dubia, cubiu } \\
\text { Solanum } \\
\text { sessiliflorum, que } \\
\text { possa proporcionar } \\
\text { redução de peso a } \\
\text { um indivíduo. }\end{array}$ & Extrato & $\begin{array}{c}\text { Instituto } \\
\text { Nacional de } \\
\text { Pesquisa da } \\
\text { Amazônia } \\
\text { (BR/AM)/Fund } \\
\text { ação } \\
\text { Universidade } \\
\text { do Amazonas } \\
\text { (BR/AM) }\end{array}$ & 2013 & $\begin{array}{l}\text { Pedido de } \\
\text { Patente }\end{array}$ \\
\hline $\begin{array}{l}\text { Composição } \\
\text { com alto valor } \\
\text { nutricional } \\
\text { baseada em } \\
\text { extratos } \\
\text { vegetais, } \\
\text { processo de } \\
\text { preparo de } \\
\text { composição e } \\
\text { método para } \\
\text { proporcional } \\
\text { nutrientes a um } \\
\text { indivíduo }\end{array}$ & $\begin{array}{c}\text { Composição com } \\
\text { alto valor } \\
\text { nutricional baseada } \\
\text { em extratos } \\
\text { vegetais, processo } \\
\text { de preparo de } \\
\text { composição e } \\
\text { método para } \\
\text { proporcionar } \\
\text { nutrientes a um } \\
\text { indivíduo }\end{array}$ & Extrato & $\begin{array}{l}\text { Instituto } \\
\text { Nacional de } \\
\text { Pesquisa da } \\
\text { Amazônia } \\
\text { (BR/AM)/ } \\
\text { Fundação } \\
\text { Universidade } \\
\text { do Amazonas } \\
\text { (BR/AM) }\end{array}$ & 2013 & $\begin{array}{l}\text { Pedido de } \\
\text { Patente }\end{array}$ \\
\hline
\end{tabular}

Fonte: Plataforma lens.org e INPI.

O uso do fruto de Myrciaria dubia está sendo usado para a produção de composição tópica natural e de dupla função para cuidados com a pele (esfoliar e hidratar) (Stewart e Asadoriana, 2018). Além de composições tópicas contendo extrato aquoso da espécie vegetal para reduzir a aparência da estria na pele (Carle et al., 2018). Quando se refere ao uso oral de produtos para fins estéticos, Smith (2009) e Parham (2017) desenvolveram formulações antienvelhecimentos. Inclusive a composição requerida por Smith (2009) também pode ser de uso tópico. 
Rico em compostos bioativos que lhe conferem capacidade antioxidante, torna-se um alimento com alta capacidade funcional, capaz de promover benefícios à saúde. Vivanco et al. (2013) desenvolveram uma composição com efeito sinérgico do extrato de Myrciaria dubia e Echinacea purpurea, a qual apresenta propriedades antioxidantes e antiinfluenza, bem como uma alta capacidade imunoestimulante. Aém disso o uso do fruto de Myrciaria dubia é usada em composições capazes de melhorar o bem-estar de uma pessoa, diminuindo os riscos de doenças cardiovasculares e/ou de Alzheimer doenças e/ou redução do açúcar no sangue usando ingredientes naturais e sintéticos (Mazed e Mazed, 2010). Não obstante, uma composição para aumentar a saúde de plantas foi reiinvidicada por Easterling (2019), a qual foi desenvolvida com uma mistura microbiana líquida e um elixir de pedra preciosa, caracterizada pelo fato de compreender ainda pelo menos um pó de frutas de camu-camu, um pó de raiz e raiz de Muira Puama e um poder de raiz de Maca.

Uma pantente concedida, reenvidica as técnicas de extração e/ou préconcentração foram reivindicadas por Pous e Navarro (2014), e Bonnlander e Kriesl (2019), em métodos para obter produtos antioxidantes da fruta Myrciaria dubia. 0 método de Pous e Navarro (2014), consite em usar fruto sem semente a temperatura inferior a $60^{\circ} \mathrm{C}$, com proporção de substâncias solúveis/acidez entre 1, 40 e 2,30 e peso de vitamina $\mathrm{C}$ entre $40 \%$ e $80 \%$ em peso de vitamina C e inclui entre $10 \%$ e $30 \%$ em peso de vitamina C glucosilada, onde a composição é uma composição alimentar, farmacêutica ou cosmética. Já o método de Plantextrakt GMBH \& Co. KG, em 2016, na INPI, e em 2019 no lens.org, foi de uma preparação de um extrato seco e pouco higroscópico de uma espécie de fruta ou vegetal rica em vitamina C, como a acerola Malpighia glabra ou camu-camu Myrciaria dubia, incluindo as etapas de preparação, remoção de açúcar, remoção de etanol e secagem.

Bebidas com Myrciaria dubia foram reinvidicadas pelo Instituto Federal de Educação, Ciência e Tecnologia de Rondônia, em 2019, sendo essas o vinho seco, vinho suave e cerveja, já o Instituto Nacional de Pesquisas da Amazônia, requeriu um nectar de baixa caloria com potencial dietético em 2013, no mesmo ano foram reivindicadas três composições baseada em extratos vegetais, a partir de frutos do cupuaçu Theobroma grandiflorum, camu-camu Myrciaria dubia, cubiu Solanum sessiliflorum, uma para redução da glicemia e/ou colesterolemia, outra para para redução de peso e a ultima para proporcionar nutrientes ao indivíduo.

\section{Desenvolvimento de produtos a partir de Myrciaria dubia: artigos científicos em base de dados}

Foram encontrados 212 artigos que mencionvam a espécie vegetal, estes dados, foram filtrados, onde só foram incluídos neste estudo os artigos que discorriam sobre o desenvolvimento de produtos (nove artigos), publicados entre 2003 e 2019. Cabe destacar que a revista que mais publicou a temática foi a Food Science and Technology (Tabela 3). Os resultados encontrados demostram um mercado promissor para o desenvolvimento de produtos a partir do fruto de Myrciaria dubia na produção de cosméticos e indústria de alimentos. Sendo assim, vários estudos estão sendo realizados, a fim de melhorar essa adesão do consumo da fruta . Dentre esses estão o estudo de Maeda et al . (2007), que demonstrou a viabilidade tecnológica e nutricional do néctar de camu -camu por ser um produto atraente de cor, sabor, aroma, aceitabilidade e como fonte de vitamina $\mathrm{C}$, tendo uma aceitabilidade global de 89,1\%.

Outra alternativa sugerida por Grigio et al. (2019), foi usar o camu-camu na fabricação de picolé, um alimento de consumo rotineiro, podendo ser transformado e explorado comercialmente como fonte natural de vitamina $\mathrm{C}$ e compostos bioativos. Em contrapartida Bardales et al. (2016), produziu hidromel, a partir da preparação de um mosto diluindo o mel com sucos de frutas do camu-camu Myrciaria dubia e do aguaymanto Physalis peruano, atribuindo capacidade antioxidante e o conteúdo de vitamina $\mathrm{C}$ no 
hidromel. Como também Vieira et al. (2010) comprovou que o fruto de camu-camu apresentou boas características físicas e químicas e alto teor de ácido ascórbico, mostrando-se viável ao processamento de licor, com $\mathrm{pH}$ baixo, alta acidez e boa aceitabilidade pelos provadores.

Tabela 3. Periódicos científicos com publicadas referente a Myriciaria dubia.

\begin{tabular}{|l|c|c|}
\hline Revista & $\begin{array}{c}\text { Ano de } \\
\text { publicação }\end{array}$ & $\begin{array}{c}\text { Qunatidade de publicações } \\
\text { por revista }\end{array}$ \\
\hline Journal of Microencapsulation & 2003 & 1 \\
\hline Food Science and Technology & $2007 / 2019$ & 2 \\
\hline Food Research International & 2011 & 1 \\
\hline Brazilian Journal of Food Technology & 2013 & 1 \\
\hline Revista de La Sociedad Química Del Perú & 2014 & 1 \\
\hline Investigación Valdizana & 2016 & 1 \\
\hline Interciencia & 2018 & 1 \\
\hline CyTA - Journal of Food & 2018 & 1 \\
\hline
\end{tabular}

Fonte: Portal Coordenação de Aperfeiçoamento de Pessoal de Nível Superior (CAPES).

Tabela 4. Publicação referentes a desenvolvimento de produtos de Myrciaria dubia no período de 2003 a 2019.

\begin{tabular}{|c|c|c|c|c|c|}
\hline Produto & $\begin{array}{l}\text { Parte da } \\
\text { planta }\end{array}$ & Classificação & Revista & Autor & Ano \\
\hline $\begin{array}{c}\text { Microencapsulação } \\
\text { do suco }\end{array}$ & $\begin{array}{c}\text { Fruto } \\
\text { congelado }\end{array}$ & Alimento & \begin{tabular}{|c|} 
Journal of \\
Microencapsulation
\end{tabular} & $\begin{array}{l}\text { Dib Taxi et } \\
\text { al. }\end{array}$ & 2003 \\
\hline $\begin{array}{l}\text { Nectar de camu- } \\
\quad \text { camu }\end{array}$ & $\begin{array}{c}\text { Fruto } \\
\text { despolpado } \\
\text { em } \\
\text { temperatura } \\
\text { ambiente e } \\
\text { sob } \\
\text { refrigeração } \\
\text { na presença e } \\
\text { ausência de } \\
\text { luz }\end{array}$ & Alimento & $\begin{array}{l}\text { Food Science and } \\
\text { Technology }\end{array}$ & $\begin{array}{l}\text { Maeda et } \\
\text { al. }\end{array}$ & 2007 \\
\hline Licor & $\begin{array}{l}\text { Os frutos sem } \\
\text { as sementes } \\
\text { foram } \\
\text { macerados } \\
\text { juntamente } \\
\text { com o álcool } \\
\text { de cereais por } \\
\text { sete dias. }\end{array}$ & Alimento & $\begin{array}{l}\text { Alimentos e } \\
\text { Nutrição }\end{array}$ & Vieira et al. & 2010 \\
\hline Suco & $\begin{array}{l}\text { Polpa Bela } \\
\text { Ischia }{ }^{\circledR}\end{array}$ & Alimento & $\begin{array}{c}\text { Food Research } \\
\text { International }\end{array}$ & $\begin{array}{l}\text { Vidigal et } \\
\text { al. }\end{array}$ & 2011 \\
\hline
\end{tabular}


Tabela 4. Continuação.

\begin{tabular}{|c|c|c|c|c|c|}
\hline Produto & $\begin{array}{c}\text { Parte da } \\
\text { planta }\end{array}$ & Classificação & Revista & Autor & Ano \\
\hline Suco clarificado & $\begin{array}{l}\text { Polpa de fruto } \\
\text { congelado }\end{array}$ & Alimento & $\begin{array}{c}\text { Brazilian Journal of } \\
\text { Food Technology }\end{array}$ & $\begin{array}{c}\text { Barreto et } \\
\text { al. }\end{array}$ & 2013 \\
\hline $\begin{array}{l}\text { Loção fotoprotetora } \\
\text { e antioxidante }\end{array}$ & $\begin{array}{c}\text { Frutos do } \\
\text { camu-camu } \\
\text { liquefeitos } \\
\text { com cubos de } \\
\text { gelo e uma } \\
\text { solução de } \\
\text { ácido cítrico } \\
(5: 1)\end{array}$ & Cosmético & $\begin{array}{c}\text { Revista de la } \\
\text { Sociedad Química } \\
\text { del Perú }\end{array}$ & $\begin{array}{c}\text { Camones et } \\
\text { al. }\end{array}$ & 2014 \\
\hline Hidromel & $\begin{array}{c}\text { Polpa de fruta } \\
\text { liquefeita }\end{array}$ & Alimento & $\begin{array}{c}\text { Investigación } \\
\text { Valdizana }\end{array}$ & $\begin{array}{c}\text { Bardales et } \\
\text { al. }\end{array}$ & 2016 \\
\hline $\begin{array}{l}\text { Cosméticos, } \\
\text { cápsulas, vinagre, } \\
\text { farinha, bebida } \\
\text { gaseificada, néctar, } \\
\text { geleia e yogurt }\end{array}$ & Polpa & $\begin{array}{l}\text { Alimento, } \\
\text { suplemento e } \\
\text { cosmético }\end{array}$ & Interciencia & $\begin{array}{l}\text { Santos et } \\
\text { al. }\end{array}$ & 2018 \\
\hline $\begin{array}{l}\text { Estabilidade da cor } \\
\text { do cordeiro moído } \\
\text { durante } \\
\text { armazenamento } \\
\text { refrigerado }\end{array}$ & $\begin{array}{l}\text { Extratos de } \\
\text { semente e } \\
\text { casca }\end{array}$ & Alimento & $\begin{array}{c}\text { Cyta - Journal of } \\
\text { Food }\end{array}$ & $\begin{array}{l}\text { Guedes- } \\
\text { Oliveira et } \\
\text { al. }\end{array}$ & 2018 \\
\hline Picolé & $\begin{array}{l}\text { Polpa sem } \\
\text { casa e } \\
\text { semente }\end{array}$ & Alimento & $\begin{array}{l}\text { Food Science and } \\
\text { Technology }\end{array}$ & Grigio et al. & 2019 \\
\hline
\end{tabular}

Fonte: Portal Coordenação de Aperfeiçoamento de Pessoal de Nível Superior (CAPES).

A fim de substituir antioxidantes artificiais, Guedes-Oliveira et al. (2018), sugere que os extratos de casca e semente de M. dubia pode ser considerada uma solução viável, a fim de melhorar a estabilidade oxidativa lipídica durante o armazenamento de cordeiros com mínimo efeito negativo nos parâmetros de cor, a fim de otimizar a aplicação de extratos e promover o desenvolvimento de produtos de cordeiro.

Dentre os produtos de Myrciaria dubia encontrados nas publicações observa-se uma variação voltada para indústria de cosmético e alimentos (Tabela 4). A produção de frutos de Myrciaria dubia para o mercado alimentício foi predomimante, devido a sua concentração de vitamina C (Yuyama, 2011).

O suco de camu-camu microencapsulado foi desenvolvido por Dib Taxi et al. (2003), em um processo para otimizar as condições operacionais, afim de melhor apresentá-lo ao mercado consumidor, de forma a garantir o seu teor de vitamina C. Bem como, Barreto et al. (2013), que avaliou o desempenho do processo de microfiltração a para obtenção de suco de camu-camu clarificado, onde o efeito da temperatura a $45{ }^{\circ} \mathrm{C}$ apresentou a melhor retenção de vitamina $\mathrm{C}$ e compostos fenólicos totais, bem como sobre o valor da atividade antioxidante total do produto obtido.

Já na no setor de cosméticos, as formulacões contendo extratos de camu-camu com ação fotoprotetora e antioxidante fornecidos pelo Laboratório Ayru Cosmetic, tiveram as taxas de irritação (II) obtidas em todas as formulações cosméticas permitem classificá-las 
como não irritantes, o que assegurou a continuidade os ensaios de eficácia clínica (Camones et al., 2014).

Uma cadeia de produção do camu-camu, nas regiões de Loreto e Ucayali em 2016, identificou o uso de produtos a partir do fruto de Myrciaria dubia (Cosméticos, cápsulas, vinagre, farinha, bebida gaseificada, néctar, geleia e yogurt), identificando-se a necessidade de formulação de políticas públicas que visem intensificar e aprimorar a interação sociotécnica entre os elos da cadeia produtiva e seus respectivos agentes (Santos et al., 2018).

\section{Produtos comercializados com Myrciaria dubia}

$\mathrm{Na}$ plataforma Google, foram encontradas 33 marcas de produtos, as mesmas formam ordenados por nome do produto, marca, apresentação e preço.

Os resultados encontrados demostram que $M$. dubia está sendo comercializado de diferentes formas no território brasileito, pois além da elevada concentração de vitamina C, estudos relatam outros compostos ativos (polifenois, carotenóides, entre outros) de interesse biológico (Fujita et al., 2015; Neves et al., 2015; Freitas et al., 2016).

Todavia, segundo Vidigal et al. (2011), apesar dos benefícios já conhecidos do fruto, o principal fator que motiva a população ao consumo do suco de frutas é o sabor, e não os benefícios para a saúde, sendo assim, a Myrciaria dubia tem um nível rejeição sensorial, devido seu sabor azedo-amargo. Embasado nisso os resultados demostram que a uma variedade de produtos derivados de camu-camu, afim de melhorar o sensorial. No que se refere aos produtos amplamante vendidos pela internet, o fruto da Myrciaria dubia é mais vendido em pó (19), cápsulas (14), licor (3), chocolate (1), geleia (1) e muda (1). Seu valor varia entre $R \$ 12,50$ e $R \$ 199,00$ reias (Tabela 5).

Tabela 5. Produtos elaborados a partir de Myrciaria dubia e comercializados.

\begin{tabular}{|c|c|c|c|c|c|}
\hline Produto & Marca & Descrição & Quant. & $\begin{array}{c}\text { Preço } \\
\text { (R\$) }\end{array}$ & Composição \\
\hline $\begin{array}{l}\text { Farinha de camu- } \\
\text { camu } 100 \mathrm{~g}\end{array}$ & $\begin{array}{l}\text { Quatro estrelas } \\
\text { empório }\end{array}$ & Pó & $100 \mathrm{~g}$ & 12,50 & Sem descrição \\
\hline $\begin{array}{l}\text { Extrato de camu- } \\
\text { camu a granel }\end{array}$ & $\begin{array}{l}\text { Armazém São } \\
\text { Vito }\end{array}$ & Pó & $200 \mathrm{~g}$ & 15,20 & Sem descrição \\
\hline $\begin{array}{l}\text { Extrato de camu- } \\
\text { camu a granel }\end{array}$ & $\begin{array}{l}\text { Armazém São } \\
\text { Vito }\end{array}$ & Pó & $500 \mathrm{~g}$ & 38,00 & Sem descrição \\
\hline $\begin{array}{c}\text { Extrato de camu- } \\
\text { camu a granel }\end{array}$ & $\begin{array}{c}\text { Armazém São } \\
\text { Vito }\end{array}$ & Pó & $1 \mathrm{~kg}$ & 76,00 & Sem descrição \\
\hline $\begin{array}{c}\text { Camu-camu em pó } \\
\text { a granel }\end{array}$ & $\begin{array}{l}\text { Empório grão de } \\
\text { ouro }\end{array}$ & Pó & $100 \mathrm{~g}$ & 19,90 & Sem descrição \\
\hline $\begin{array}{c}\text { Camu-camu } \\
\text { concentrado } \\
\text { solúvel supraervas } \\
150 \mathrm{~g}\end{array}$ & SupraErvas & Pó & $150 \mathrm{~g}$ & 26,38 & $\begin{array}{l}\text { Polidextrose, } \\
\text { camu-camu } \\
\text { solúvel, } \\
\text { antiumectante } \\
\text { dióxido de silício, } \\
\text { aroma idêntico } \\
\text { ao natural, e } \\
\text { edulcorante } \\
\text { natural } \\
\text { glicosídeos de } \\
\text { esteviol (stévia). }\end{array}$ \\
\hline
\end{tabular}

Rev. Bras. Gest. Amb. Sustent., 2021, vol. 8, n. 20, p. 1687-1706. 
Tabela 5. Continuação.

\begin{tabular}{|c|c|c|c|c|c|}
\hline Produto & Marca & Descrição & Quant. & $\begin{array}{c}\text { Preço } \\
\text { (R\$) }\end{array}$ & Composição \\
\hline $\begin{array}{l}\text { Camu-camu em pó } \\
\text { tui alimentos } 150 \mathrm{~g}\end{array}$ & TUI alimentos & Pó & $150 \mathrm{~g}$ & 26,95 & Sem descrição \\
\hline $\begin{array}{c}\text { Grão granola } \\
\text { beterraba + camu- } \\
\text { camu e açaí } 200 \mathrm{~g}\end{array}$ & Selections & pó & $200 \mathrm{~g}$ & 29,90 & $\begin{array}{c}\text { Flocos de milho, } \\
\text { semente de } \\
\text { girassol, flocos de } \\
\text { arroz, } \\
\text { concentrado de } \\
\text { maçã em pó, } \\
\text { painço, grão de } \\
\text { bico, linhaça } \\
\text { dourada, flocos } \\
\text { de ervilha, } \\
\text { beterraba em pó, } \\
\text { extrato seco de } \\
\text { camu-camu, açaí } \\
\text { em pó e } \\
\text { antioxidante } \\
\text { tocoferol. }\end{array}$ \\
\hline $\begin{array}{l}\text { Camu-camu em pó } \\
\text { cool dried } 100 \mathrm{~g}\end{array}$ & Ynadú & Pó & $100 \mathrm{~g}$ & 39,00 & Sem descrição \\
\hline $\begin{array}{l}\text { Camu-camu em pó } \\
500 \text { g levnutra }\end{array}$ & $\begin{array}{l}\text { Levnutra } \\
\text { Produtos } \\
\text { Naturais e } \\
\text { Orgânicos }\end{array}$ & Pó & $500 \mathrm{~g}$ & 41,00 & $\begin{array}{c}\text { Suco de camu- } \\
\text { camu } \\
\text { desidratado, } \\
\text { maltodextrina, } \\
\text { antioxidante } \\
\text { INS300 e corante } \\
\text { INS120. }\end{array}$ \\
\hline $\begin{array}{c}\text { Camu-camu em pó } \\
\text { souly } 60 \mathrm{~g}\end{array}$ & Souly & Pó & $60 \mathrm{~g}$ & 48,90 & $\begin{array}{l}\text { Camu-camu em } \\
\text { pó }\end{array}$ \\
\hline $\begin{array}{c}\text { Camu-camu } \\
\text { puro reposição } \\
\text { vitamina C } 300 \mathrm{~g} \\
\text { produto puro*** }\end{array}$ & Saúde Naturalfc & Pó & $300 \mathrm{~g}$ & 53,90 & Sem descrição \\
\hline $\begin{array}{c}\text { Farinha de camu- } \\
\text { camu - liofilizado } \\
\text { em pó - } 500 \text { g com } \\
\text { laudo }\end{array}$ & Selettogrão & Pó & $500 \mathrm{~g}$ & 69,90 & Sem descrição \\
\hline $\begin{array}{c}\text { Pó camu-camu, } \\
99 \mathrm{~g}\end{array}$ & Wilderness Poets & Pó & $99 \mathrm{~g}$ & 82,04 & $\begin{array}{c}\text { Orgânico, cru, } \\
\text { camu-camu berry } \\
\text { em pó }\end{array}$ \\
\hline $\begin{array}{c}\text { Pó orgânico camu- } \\
\text { camu - } 3.5 \text { oz. }\end{array}$ & Zint & Pó & $99 \mathrm{~g}$ & 83,62 & $\begin{array}{c}\text { Pó de camu-camu } \\
\text { orgânico }\end{array}$ \\
\hline $\begin{array}{l}\text { Pó camu-camu } \\
100 \mathrm{~g} .-3.5 \mathrm{oz} .\end{array}$ & $\begin{array}{c}\text { Sunfood } \\
\text { Superfoods }\end{array}$ & Pó & $100 \mathrm{~g}$ & 126,93 & $\begin{array}{c}\text { Camu-camu cru } \\
\text { orgânico } \\
\text { certificado. } \\
\end{array}$ \\
\hline $\begin{array}{c}\text { Pó camu-camu - } \\
85 \mathrm{~g}\end{array}$ & Nativas organics & Pó & $85 \mathrm{~g}$ & 137,51 & $\begin{array}{c}\text { Pó orgânico } \\
\text { certificado de } \\
\text { camu-camu } \\
\text { (Myciaria dubia) }\end{array}$ \\
\hline
\end{tabular}


Tabela 5. Continuação.

\begin{tabular}{|c|c|c|c|c|c|}
\hline Produto & Marca & Descrição & Quant. & $\begin{array}{c}\text { Preço } \\
\text { (R\$) }\end{array}$ & Composição \\
\hline $\begin{array}{l}\text { Camu-camu em pó } \\
\text { terramazonia } 1 \mathrm{~kg}\end{array}$ & Terramazonia & Pó & $1 \mathrm{~kg}$ & 150,00 & $\begin{array}{l}\text { polpa de camu- } \\
\text { camu e } \\
\text { maltodextrina }\end{array}$ \\
\hline $\begin{array}{c}\text { Camu-camu } 100 \% \\
\text { natural e em pó } \\
1 \mathrm{~kg}\end{array}$ & $\begin{array}{l}\text { Cerealista } \\
\text { Express }\end{array}$ & Pó & $1 \mathrm{~kg}$ & 199,90 & Sem descrição \\
\hline $\begin{array}{l}\text { Camu-camu - } 60 \\
\text { cápsulas - Bioklein }\end{array}$ & Bioklein & Cápsulas & $500 \mathrm{mg}$ & 16,91 & $\begin{array}{l}\text { Fruto do camu- } \\
\text { camu em pó. } \\
\text { Excipiente Talco } \\
\text { Pharma. } \\
\text { Composição da } \\
\text { Cápsula: Gelatina. } \\
\text { Não contém } \\
\text { glúten } \\
\end{array}$ \\
\hline $\begin{array}{c}\text { Camu-camu unilife } \\
60 \text { cápsulas de } \\
500 \mathrm{mg}\end{array}$ & Unilife & Cápsulas & $500 \mathrm{mg}$ & 19,94 & $\begin{array}{l}\text { Conteúdo: Camu- } \\
\text { camu em pó. } \\
\text { Cápsulas: } \\
\text { gelatina } \\
\text { vegetariana e } \\
\text { água. } \\
\end{array}$ \\
\hline $\begin{array}{c}\text { Camu-camu + } \\
\text { Vitamina C } 100 \% \\
\text { natural imunidade }\end{array}$ & REI Terra & Cápsulas & $500 \mathrm{mg}$ & 21,01 & $\begin{array}{l}\text { Camu-camu em } \\
\text { pó, vitamina C. } \\
\text { Composição da } \\
\text { cápsula: gelatina } \\
\text { e umectante } \\
\text { glicerina } \\
\end{array}$ \\
\hline $\begin{array}{l}\text { Camu-camu - } 60 \\
\text { cápsulas - } \\
\text { Maxinutri }\end{array}$ & Maxiutri & Cápsulas & $400 \mathrm{mg}$ & 21,90 & $\begin{array}{c}\text { Camu-camu } \\
\text { (Myrciaria dubia) } \\
\text { em pó, } \\
\text { excipiente: } \\
\text { silicato de } \\
\text { magnésio, } \\
\text { antiumectante: } \\
\text { dióxido de silício, } \\
\text { cápsula: gelatina. } \\
\text { Não contém } \\
\text { glúten. }\end{array}$ \\
\hline $\begin{array}{l}\text { Camu-camu - } 60 \\
\text { cápsulas - Promel }\end{array}$ & Promel & Cápsulas & $500 \mathrm{mg}$ & 22,90 & $\begin{array}{l}\text { Camu-camu em } \\
\text { pó e } \\
\text { maltodextrina. } \\
\text { Composição da } \\
\text { cápsula: Gelatina } \\
\text { e umectante } \\
\text { glicerina. Não } \\
\text { contém glúten. }\end{array}$ \\
\hline $\begin{array}{l}\text { Super vitamina C - } \\
\text { acelora, laranja e } \\
\text { camu-camu } 120 \\
\text { cápsulas } 500 \mathrm{mg}\end{array}$ & REI Terra & Cápsulas & $500 \mathrm{mg}$ & 27,90 & $\begin{array}{c}\text { Acerola em pó, } \\
\text { Camu-camu, } \\
\text { Laranja em pó e } \\
\text { amido de milho }\end{array}$ \\
\hline
\end{tabular}


Tabela 5. Continuação.

\begin{tabular}{|c|c|c|c|c|c|}
\hline Produto & Marca & Descrição & Quant. & $\begin{array}{c}\text { Preço } \\
\text { (R\$) }\end{array}$ & Composição \\
\hline $\begin{array}{l}\text { Camu-camu para } \\
\text { imunidade } 60 \\
\text { cápsulas } 500 \mathrm{mg}\end{array}$ & Melcoprol & Cápsulas & $500 \mathrm{mg}$ & 34,00 & Sem descrição \\
\hline $\begin{array}{c}\text { Camu-camu } \\
\text { vitamina C } \\
500 \mathrm{mg} \text { fauna e } \\
\text { flora } 60 \text { cápsulas }\end{array}$ & Fauna E Flora & Cápsulas & $500 \mathrm{mg}$ & 37,00 & Sem descrição \\
\hline $\begin{array}{l}\text { Camu-camu } 100 \\
\text { cápsulas 500mg - } \\
\text { vitamina C - } \\
\text { Amazom Life }\end{array}$ & Frutas Exóticas & Cápsulas & $500 \mathrm{mg}$ & 38,00 & Sem descrição \\
\hline $\begin{array}{l}\text { Camu-camu - } 60 \\
\text { cápsulas } 250 \text { mg }\end{array}$ & PhytosQuallys & Cápsulas & $250 \mathrm{mg}$ & 70,00 & $\begin{array}{c}\text { Camu-camu } \\
\text { (Myrciaria dubia } \\
\text { H. B. Kunth), } \\
\text { Dióxido de Silício } \\
\text { (Antiumectante). } \\
\text { Invólucro: } \\
\text { Gelatina e água } \\
\text { purificada. Não } \\
\text { contém glúten. }\end{array}$ \\
\hline $\begin{array}{c}\text { Paradise herbs, } \\
\text { camu-camu - } 60 \\
\text { cápsulas } \\
\text { vegetarianas } \\
\end{array}$ & Paradise & Cápsulas & $400 \mathrm{mg}$ & 74,70 & Sem descrição \\
\hline $\begin{array}{c}\text { Amazon } \\
\text { Therapeutic Labs, } \\
\text { camu-camu - } 60 \\
\text { cápsulas }\end{array}$ & $\begin{array}{c}\text { Amazon } \\
\text { Therapeutic Labs }\end{array}$ & Cápsulas & $500 \mathrm{mg}$ & 122,18 & Sem descrição \\
\hline $\begin{array}{l}\text { Extrato de camu- } \\
\text { camu, } 2000 \mathrm{mg}, \\
120 \text { cápsulas, } 2 \\
\text { frascos }\end{array}$ & Piping Rock & Cápsulas & $2 \mathrm{~g}$ & 122,45 & $\begin{array}{c}\text { Camu-camu, } \\
\text { cápsula de } \\
\text { gelatina, Pó de } \\
\text { arroz, Estearato } \\
\text { de magnésio } \\
\text { vegetal, Sílica } \\
\end{array}$ \\
\hline $\begin{array}{c}\text { Kit camu-camu } 500 \\
\text { mg } 60 \text { cápsulas } 6 \\
\text { potes premium }\end{array}$ & Fitoplant & Cápsulas & $500 \mathrm{mg}$ & 171,00 & Sem descrição \\
\hline $\begin{array}{l}\text { Licor de camu- } \\
\text { camu } 275 \mathrm{~mL}\end{array}$ & $\begin{array}{c}\text { Deveras } \\
\text { Amazônia }\end{array}$ & Licor & $275 \mathrm{~mL}$ & 28,00 & Sem descrição \\
\hline $\begin{array}{c}\text { Licor de camu- } \\
\text { camu }\end{array}$ & $\begin{array}{c}\text { Tacaburi Furtos } \\
\text { da Amazônia }\end{array}$ & Licor & $250 \mathrm{~mL}$ & 20,00 & Sem descrição \\
\hline $\begin{array}{l}\text { Licor de camu- } \\
\text { camu }\end{array}$ & $\begin{array}{c}\text { Tacaburi Furtos } \\
\text { da Amazônia }\end{array}$ & Licor & $500 \mathrm{~mL}$ & 30,00 & Sem descrição \\
\hline
\end{tabular}


Tabela 5. Continuação.

\begin{tabular}{|c|c|c|c|c|c|}
\hline Produto & Marca & Descrição & Quant. & $\begin{array}{c}\text { Preço } \\
\text { (R\$) }\end{array}$ & Composição \\
\hline $\begin{array}{c}\text { Chocolate zero } \\
\text { açúcar amargo em } \\
\text { tablete } 67 \% \text { cacau } \\
\text { superfoods sabor } \\
\text { baru e camu-camu } \\
80 \mathrm{~g}\end{array}$ & Superfoods & Chocolate & $80 \mathrm{~g}$ & 26,99 & $\begin{array}{c}\text { massa de cacau, } \\
\text { SNew® (eritritol, } \\
\text { fibra de milho } \\
\text { não transgênico e } \\
\text { estévia), } \\
\text { manteiga de } \\
\text { cacau, cacau em } \\
\text { pó natural, } \\
\text { castanha do Baru, } \\
\text { farinha de } \\
\text { mesocarpo de } \\
\text { babaçu, camu- } \\
\text { camu em pó e } \\
\text { emulsificantes: } \\
\text { lecitina de } \\
\text { girassol e } \\
\text { polirricinoleato } \\
\text { de poliglicerila. } \\
\text { Não contém } \\
\text { glúten. }\end{array}$ \\
\hline $\begin{array}{l}\text { Geléia de camu- } \\
\text { camu (150 g) }\end{array}$ & $\begin{array}{c}\text { Deveras } \\
\text { Amazônia }\end{array}$ & Geléia & $150 \mathrm{~g}$ & 22,00 & Sem descrição \\
\hline $\begin{array}{c}\text { Camu-camu - fruta } \\
\text { c/ o segundo maior } \\
\text { teor de vitamina C } \\
\text { do mundo }\end{array}$ & Jardim Exótico & Muda & 1 & 39,80 & Sem descrição \\
\hline
\end{tabular}

Fonte: Busca livre no Google.

0 mercado de produtos do camu-camu mostra-se crescente e com grande potencial econômico capaz de colocá-lo no mesmo nível de importância de outras frutas tradicionais da região amazônica, como o açaí e o cupuaçu. Apesar de dificilmente serem consumidos in natura, devido à elevada acidez e o amargor da casca, os frutos são tradicionalmente empregados na fabricação de sucos, sorvetes, bebidas e geleias (Viera, 2010).

\section{Conclusão}

0 mercado com o fruto de Myrciaria dubia é diversificado. Possui pedidos de patentes e patentes na área de cosmetologia e bebibas, além de diversos artigos que contribuem para seu potencial mercadológico como alimento/suplemento e cosmético, o que otimiza a sua produção no comércio nas mais diversas formas de produção, hidromel, suco, licor, néctar, picolé, cápsulas, extratos, formulações cosméticas entre outros.

O fruto do camucamuzeiro possui compostos bioativos (polifenois, ácido ascórbico, protoancioninas) capazes de produzir efeitos biológicos como antioxidantes, fotoprotetoras, inunoestimulante, antiinfluenza, usados para o tratamento de doenças cardiovasculares, Alzheimer e redução da glicemia, além de uso estético no antienvelhecimento e estrias. Portanto, pode-se concluir que produtos oriundos do fruto de Myrciaria dubia estão incluídos na rotina do consumidor, o qual traz benefícios na área da indústria de alimentos, cosmético e farmacêutica, sendo um produto promissor ao 
mercado e a agroindústria pelo seu potencial tecnológico e nutricional, o que pode trazer grandes avanços para o aproveitamento das fruteiras amazônicas com possibilidade de aproveitamento industrial, possibilitando a criação de empregos.

\section{Conflito de interesses}

As autoras declaram não haver conflito de interesses.

\section{Referências}

ANVISA - Agência Nacional de Vigilância Sanitária. Resolução RDC no 17, de 16 de abril de 2010. Dispõe sobre as boas práticas de fabricação de medicamentos. Disponível em: <https://bvsms.saude.gov.br/bvs/saudelegis/anvisa/2010/res0017_16_04_2010.html>. Acesso em: 14 jul. 2021.

Arruda, A.; lima, R.; Silva, R.; Peixoto, N. Desenvolvimento do camu-camu (Myrciaria dubia) em diferentes substratos nas condições de Ipameri-GO. Enciclopédia Biosfera, v. 7, n. 12, p. 1-7, 2011.

Bardales, A. D. N.; Portal, R. M. R.; Garay, S. G. M. Determinación de las propiedades funcionales y sensoriales del hidromiel elaborado con camu-camu (Myrciaria dubia) y aguaymanto (Physalis peruviana). Investigación Valdizana, v. 10, n. 4, p. 161-170, 2016.

Barreto, A. G.; Cabral, L. M. C.; Matta, V. M.; Freitas, S. P. Clarificação de polpa de camu-camu por microfiltração. Brazilian Journal of Food Technology, v. 16, n. 3, p. 207$215,2013$.

Billacrês, M. A. R.; Costa, R. C.; Nunez, C. V. A cadeia produtiva na Gestão da Inovação da Biotecnologia: o camu-camu (Myrciaria dubia HBK) no Amazonas. Revista Fitos, v. 14, n. 2, p. 222-237, 2020. https://doi.org/10.32712/2446-4775.2020.1077

Bonnländer, B.; Kriesl, E. Method for the preparation of a low-hygroscopic, dried extract of a vitamin C rich fruit or vegetable species. Titular: Plantextrakt GMBH \& Co. KG. EP2868208-B1. Depósito: 31 out. 2013. Concessão: 17 abr. 2019.

Botto, J.-M.; Capallere, C.; Gondran, C.; Imbert, I.; Garnier, S.; Le Borgne, E. Myrciaria dubia fruit extracts rich in organic acids, cosmetic compositions comprising same and cosmetic uses there of. Titular: ISP Investments LLC; Jafer Enterprises R\&D S L Soc Unipersonal. W02019/149864-A1. Depósito: 31 jan. 2019. Concessão: 08 ago. 2019.

Camones, M. A. I.; Chota, G. E. T.; Malla, J. H.; Jáuregui, A. M. M.; Morán, R. I. G.; Fuentes, G. Q.; Pacheco, C. J. P.; Espinoza, E. D. R. T. Actividad antioxidante y fotoprotectora in vitro de uma loción y gel elaborados con extracto estabilizado de camu-camu (Myrciaria dubia kunth.). Revista de La Sociedad Química Del Perú, v. 80, n. 1, p. 65-77, 2014.

Carle, T.; Kalahasti, G.; Burkes, S.; Gan, D. Methods and compositions for treating striae distensae. Titular: Mary Kay Inc. US2018/0092955-A1. Depósito: 04 out. 2017. Concessão: 05 abr. 2018.

Chagas, E. A.; Bacelar-Lima, C. G.; Carvalho, A. S.; Ribeiro, M. I. G.; Sakazaki, R. T.; Neves, L. C. Propagação do camu-camu (Myrciaria dubia (HBK) McVaugh). Revista Agro@mbiente On-line, v. 6, n. 1, p. 67-73, 2012. https://doi.org/10.18227/1982-8470ragro.v6i1.634

Chagas, E. A.; Lozano, R. M. B.; Chagas, P. C.; Bacelar-Lima, C. G.; Garcia, M. I. R.; Oliveira, J. V.; Souza, O. M.; Morais, B. S.; Araújo, M. C. R. Intraspecific variability of camu-camu fruit in native populations of Northern Amazonia. Crop Breeding and Applied Biotechnology, v. 15, n. 4, p. 265-271, 2015. https://doi.org/10.1590/1984-70332015v15n4a44 
Dib Taxi, C. M. A.; Menzes, H. C.; Santos, A. B.; Grosso, C. R. F. Study of the microencapsulation of camu-camu (Myrciaria dubia) juice. Journal of Microencapsulation, $\quad$ v. 20, $\quad$ n. 4, $\quad$ p. 443-448, 2003. https://doi.org/10.1080/ 0265204021000060291

Easterling, J. H. Plant enhancing composition. Titular: Easterling John Harvey. US2019/0225555-A1. Depósito: 24 jan. 2019. Concessão: 25 maio 2019.

Freitas, C. A. B.; Silva, A. B.; Alves, C. N.; Nascimento, W. M. O.; Lopes, A. S.; Lima, M. O.; Müller, R. C. S. Characterization of the fruit pulp of camu-camu (Myrciaria dubia) of seven different genotypes and their rankings using statistical methods PCA and HCA. Journal of the Brazilian Chemical Society, v. 27, p.1838-1846, 2016. https://doi.org/10.5935/ 0103-5053.20160067

Fujita, A.; Sarkar, D.; Wu, S.; Kennelly, E.; She, K. Evaluation of phenolic-linked bioactives of camu-camu (Myrciaria dubia MC. Vaugh) for antihyperglycemia, antihypertension, antimicrobial properties and cellular rejuvenation. Food Research International, v. 77, n. 2, p. 194-203, 2015. https://doi.org/10.1016/j.foodres.2015.07.009

Garcia, R. Internacionalização comercial e produtiva na indústria de cosméticos: desafios competitivos para empresas brasileiras. Production, v. 15, n. 2, p. 158-171, 2005. https://doi.org/10.1590/S0103-65132005000200003

Grigio, M. L.; Durigan, M. F. B.; Chagas, E. A. Different formulations of camu-camu popsicle: Characterization, vitamin $\mathrm{C}$ and sensorial analysis of an opportunity to family agroindustry. Food Science and Technology, v. 39, n. 1, p.93-97, 2019. https://doi.org/10.1590/fst.38417

Grigio, M. L.; Moura, E. A.; Carvalho, G. F.; Zanchetta, J. J.; Chagas, P. C.; Chagas, E. A.; Durigan, M. F. B. Nutraceutical potential, quality and sensory evaluation of camu-camu pure and mixed jelly. Food Science and Technology, 2021. https://doi.org/10.1590/ fst.03421

Guedes-Oliveira, J. M.; Costa-Lima, B. R. C.; Cunha, L. C. M.; Salim, A. P. A. A.; Baltar, J. D.; Fortunato, A. R.; Conte-Junior, C. A. Impact of Myrciaria dubia peel and seed extracts on oxidation process and colour stability of ground lamb. CyTA - Journal of Food, v. 16, n. 1, p. 931-937, 2018. https://doi.org/10.1080/19476337.2018.1512529

INPI - Instituto Nacional da Propriedade Industrial. 2021. Disponível em: <https://busca.inpi.gov.br/pePI/jsp/patentes/>. Acesso em: 14 jul. 2021.

Hines, M.; Florence, T. Topical skin care formulations. Titular: Mary Kay Inc. US2015/0374769-A1. Depósito: 03 mar. 2015. Concessão: 31 dez. 2016.

Kahaner, L. Competitive intelligence: How to gather, analyze, and use information to move your business to the top. New York: Touchstone, 1996.

Maeda, R. N.; Pantoja, L.; Yuyama, L. K. O.; Chaar, J. M. Estabilidade de ácido ascórbico e antocianinas em néctar de camu-camu (Myrciaria dubia (H. B. K.) McVaugh). Food Science and Technology, v. 27, n. 2, p. 313-316, 2007. https://doi.org/10.1590/S010120612007000200018

Mazed, M. A.; Mazed, S. Nutritional supplement for the prevention of cardiovascular disease, Alzheimer's disease, diabetes, and regulation and reduction of blood sugar and insulin resistance. Titular: Mazed Mohammad A \& Mazed Sayeeda. US2010/0021533-A1. Depósito: 02 out. 2009. Concessão: 28 jan. 2010. 
Morais, L. C.; Pinheiro, S. S. Nutrients and bioactive compounds of açai, bacuri, buriti, camu-camu, and cubiu. In: Todorov, S. D.; Pieri, F. A. (Orgs.). Tropical fruits: From cultivation to consumption and health benefits, fruits from the Amazon. New York: Nova Science Publishers, 2018. p. 121-132.

Nagamine, K.; Hayashi, M.; Furukawa, K. Compound, process for producing the same and use thereof. Titular: Nichirei Biosciences Inc. US7074907-B2. Depósito: 29 jul. 2005. Concessão: 11 jul. 2006.

Nagamine, K.; Hayashi, M.; Furukawa, K. Whitening agent, skin preparation for external use and cosmetic. Titular: Nichirei Biosciences Inc. EP1582195-B1. Depósito: 08 dez. 2003. Concessão: 14 out. 2010.

Neves, L. C.; Siva, V. X.; Pontis, J. A.; Flach, A.; Roberto, S. R. Bioactive compounds and antioxidant activity in pre-harvest camu-camu [Myrciaria dubia (HBK) McVaugh] fruits. Scientia Horticulturae, v. 186, p. 223-229, 2015. https://doi.org/10.1016/ j.scienta.2015.02.031

Parham, M. T. Composition for an anti-aging treatment. Titular: Parham Tabibian MD Inc. US9839604-B1. Depósito: 11 nov. 2014. Concessão: 12 dez. 2017.

Pous, G. I.; Navarro, S. M. Antioxidant compositions of a product obtained from the camu-camu fruit. Titular: San Juan Amazonia Europa S L. W02014/020219-A1. Depósito: 30 jul. 2013. Concessão: 06 fev. 2014.

Ribeiro, G. D. Fruticultura tropical: uma alternativa para a agricultura de Rondônia. Porto Velho: EMBRAPA, 2006.

Rodrigues, R. B.; Marx, F. Camu-camu [Myrciaria dubia (HBK) McVaugh]: A promising fruit from the Amazon Basin. Nutrition, v. 30, n. 9, p. 376, 2006.

Santos, R. M. B.; Chagas, P. C.; Rocha, J. H. M. V; Chagas, E. A.; Panduro, M. H. P.; Lozano, R. M. B.; Rodriguez, C. A. Cadeia de produção do camu-camu (Myrciaria dubia (Kunth) McVaugh): o caso das regiões produtoras de Loreto e Ucayali na Amazônia Peruana. Interciencia, v. 43, n. 4, p. 261-268, 2018.

Smith, W. P. Methods and compositions for reducing the appearance of dynamic facial wrinkles. Titular: Smith Walter P. US2009/0104174-A1. Depósito: 25 jun. 2008. Concessão: 23 abr. 2009.

Sobral, A. T. M.; Chagas, E. A.; Andrade, J. K. C.; Santos, V. A.; Lozano, R. M. B. Enraizamento de estacas de diferentes populações de camu-camu em câmara de subirrigação. Anais do XIII Encontro do Programa de Iniciação Científica, Boa Vista, UFRR, 2014.

Stewart, L.; Asadorian, A. Topical skin care formulations and methods of using same. Titular: Stewart Lisa \& Asadorian Ara. US9999588-B1. Depósito: 16 fev. 2016. Concessão: 19 jun. 2018.

Vidigal, M. C. T. R.; Minim, V. P. R.; Carvalho, N. B.; Milagres, M. P.; Gonçalves, A. C. A. Effect of a health claim on consumer acceptance of exotic Brazilian fruit juices: Açaí (Euterpe oleracea Mart.), camu-camu (Myrciaria dubia), cajá (Spondias lutea L.) and umbu (Spondias tuberosa Arruda). Food Research International, v. 44, n. 7, p. 1988-1996, 2011. https://doi.org/10.1016/j.foodres.2010.11.028 
Viégas, I. J. M.; Thomaz, M. A. A.; Silva, J. F.; Conceição, H. E. O.; Naiff, A. P. M. Efeito da omissão de macronutrientes e boro no crescimento, nos sintomas de deficiências nutricionais e na composição mineral de plantas de camucamuzeiro. Revista Brasileira de Fruticultura, v. 26, n. 2, p. 315-319, 2004. https://doi.org/10.1590/S010029452004000200032

Viera, V. B.; Rodrigues, J. B.; Brasil, C. C. B.; Rosa, C. S. Produção, caracterização e aceitabilidade de licor de camu-camu (Myrciaria dubia (H.B.K.) McVaugh). Alimentos e Nutrição, v. 21, n. 4, p. 519-522, 2010.

Vivanco, R. F. C.; Bejarano, E. R. A. Natural antioxidant anti-influenza composition. Titular: Pharmabrand S A. W02013/029634-A2. Depósito: 31 ago. 2012. Concessão: 07 mar. 2013.

Yuyama, K. A cultura de camu-camu no Brasil. Revista Brasileira de Floricultura, v. 33, n. 2, p. 3-4, 2011. https://doi.org/10.1590/S0100-29452011000200001

(CC) Informação da Licença: Este é um artigo Open Access distribuído sob os termos da Licença Creative Commons Attribution, que permite uso irrestrito, distribuição e reprodução em qualquer meio, desde que a obra original seja devidamente citada. 